\title{
Integrating Virtual Agents in BCI Neurofeedback Systems
}

\section{- invited talk -}

\author{
Marc Cavazza, Fred Charles, \\ Stephen W. Gilroy, Julie Porteous, \\ Gabor Aranyi \\ School of Computing \\ Teesside University \\ Middlesbrough, United Kingdom \\ m.o.cavazza@tees.ac.uk
}

\author{
Gal Raz ${ }^{1,2}$, Nimrod Jakob Keynan ${ }^{1,2}$, Avihay \\ Cohen $^{1}$, Gilan Jackont ${ }^{1,2}$, Yael Jacob ${ }^{1,2}$, Eyal \\ Soreq $^{1,2}$, Ilana Klovatch ${ }^{1}$, Talma Hendler ${ }^{1,2}$ \\ ${ }^{1}$ Functional Brain Center, Tel Aviv Sourasky \\ Medical Center, Tel Aviv, Israel \\ ${ }^{2}$ Tel Aviv University, Tel Aviv, Israel \\ hendlert@gmail.com
}

\begin{abstract}
The recent development of Brain-Computer Interfaces (BCI) to Virtual World has resulted in a growing interest in realistic visual feedback. In this paper, we investigate the potential role of Virtual Agents in neurofeedback systems, which constitute an important paradigm for BCI. We discuss the potential impact of virtual agents on some important determinants of neurofeedback in the context of affective BCI. Throughout the paper, we illustrate our presentation with two fully implemented neurofeedback prototypes featuring virtual agents: the first is an interactive narrative in which the user empathises with the character through neurofeedback; the second recreates a natural environment in which crowd behaviour becomes a metaphor for arousal and the user engages in emotional regulation.
\end{abstract}

\section{Categories and Subject Descriptors}

H.5.2 [Information Interfaces and Presentation]: User Interfaces

\section{General Terms}

Human Factors; Design; Measurement

\section{Keywords}

Brain-Computer Interfaces, Neurofeedback, Virtual Reality, Affective Computing

\section{INTRODUCTION}

In recent years, we have seen a development of synergies between Brain-Computer Interfaces (BCI) and Virtual Reality applications. This has manifested itself in different ways, from the integration of BCI as an interface to virtual environments [18] and the incorporation of $\mathrm{BCI}$ in computer games [23], to the development of serious games as part of BCI-based therapeutic interventions $[20,31]$. Virtual agents have become an important component of all the above environments, for instance in computer games and interactive narratives [30], with their ability to express and elicit various types of emotions. This makes them of particular relevance to affective $\mathrm{BCI}$ [27].

In this paper, we investigate the role of virtual agents in a specific type of affective BCI system, based on neurofeedback (NF). Our

Permission to make digital or hard copies of all or part of this work for personal or classroom use is granted without fee provided that copies are not made or distributed for profit or commercial advantage and that copies bear this notice and the full citation on the first page. To copy otherwise, or republish, to post on servers or to redistribute to lists, requires prior specific permission and/or a fee.

Laval Virtual VRIC '14, April 9-11, 2014, Laval, France.

Copyright 2014 978-1-4503-2626-1 ...\$10.00 objective is to determine how virtual agents can be integrated into neurofeedback systems in a way that improves usability of NF systems. Previous research has suggested that the type of visual channel used could have an influence on user experience and NF performance [16]. In the case of affective BCI, the use of virtual agents would provide a more semantic approach to the feedback channel due to their ability to elicit emotional or social responses.

After a brief introduction to neurofeedback, and a discussion of relevant previous work, we introduce the two main dimensions of virtual agents: their ability to enhance the subject experience and their influence on the behaviour of neurofeedback loops. We then present two fully-implemented neurofeedback experiments featuring virtual agents. The first one implements an empathic BCI within an interactive narrative and the second one uses crowd behaviour as a feedback channel in an emotion regulation application.

\section{PRINCIPLES OF NEUROFEEDBACK}

Neurofeedback is the process by which subjects learn to control the activation of specific brain regions in a closed-loop setting, where they are presented with a measure of that region's activation. Recent progress has provided more rigorous foundations to neurofeedback [13], which has been proposed for performance enhancement [28] and as a therapy for several conditions [29], including mood disorders [2] and ADHD [20].

Neurofeedback has also been introduced as one possible mechanism for BCI as it provides a more stable input than other measures, and preserves the user's agency. Further, its feedback component can naturally be integrated into the interface, in particular as it is most often visual.

A neurofeedback system can be described through several essential components. The first one consists in the target brain area to be regulated. It is generally identified through its support for specific (e.g. executive) functions, or its role in cognitive processes. The second is the sensing device, which captures that region's activation [36], based on various techniques of different spatio-temporal resolutions (EEG, fMRI, fNIRS...). The third one is the feedback channel, which translates the neural activity measured by the sensor into real-time information for the user. The feedback channel has as its objective the reward of desired behaviour, by notifying the user when the target region's activity is within the target range. Another important element of any neurofeedback experiment consists in the cognitive strategies that the user will apply to control the activation of the target region $[17,28]$. These strategies account for the fact that neurofeedback aims precisely to bring under conspicuous control neural structures that are ordinarily not amenable to it. It is customary to 
differentiate between explicit and implicit strategies [33]: the former provide guidance to the user on thought contents that may result in activating the target region, while the latter leave the discovery to the user.

The development of multimedia systems and VR has influenced the design of neurofeedback systems, which can take advantage of the realistic and sophisticated nature of the display to improve visual feedback (see [33] for some examples). Importantly, this leads to embedding visual feedback into the neurofeedback application itself, thereby strengthening the feedback loop. The use of virtual agents falls into this category and will be developed in the next sections.

\section{PREVIOUS AND RELATED WORK}

The type of visual feedback used in neurofeedback systems tends to differ depending on the context of use and nature of the system. We can distinguish three main contexts: fundamental research in neuroscience, clinical applications of neurofeedback, and use of neurofeedback to support BCI applications. Research in neurofeedback has traditionally used basic visual (or aural) modalities for feedback, possibly because the need to engage users was felt less strongly with volunteers than patients.

Several authors have explored the use of NF systems as a therapy for $\mathrm{ADHD}$, and many prototypes have been developed, of increasing complexity. Since these systems target children there has been additional consideration into the acceptability and compelling nature of the visual interface [20]. Independently of the generic interest in serious gaming, the familiarity of user population with computer games made them a natural medium to develop neurofeedback systems. A neurofeedback system for ADHD therapy has been developed as part of the OpenVIBE project [18]. In this system, the user is immersed in a regular classroom in first-person mode, populated with virtual characters representing other pupils. The user's perspective is one of sitting at their desk in a normal classroom, facing the blackboard. Neurofeedback takes place through two main mechanisms. The first one consists in blurring the image of a virtual TV screen situated inside the classroom. However, the main feedback mechanism consists in seeing supplies on the pupil's desk levitating when the user fails to meet the activity threshold. The visual feedback hence consists in the intensity of this levitation, which is equivalent to the motion parameters of a virtual agent. The fact that objects rather than characters form the agent population does not impair efficiency, and the use of the environment itself as an agent for narrative purposes has been previously reported [22].

The third category consists in BCI that have been specifically developed for multimedia applications and therefore inherit the visual richness of the embedding application. One particular case is constituted by BCI to computer games, which have attracted significant interest in recent years [23]. For instance, in "alpha World of Warcraft" [4], the level of alpha activation detected from the user is used to transform the appearance of the player character between one of the in-game categories.

Finally, Jensen et al. [16] conducted one of the first specific studies on the impact of visual feedback in neurofeedback applications from an engineering perspective. They compared different levels of sophistication and integrative properties of the visual interface on a task of alpha-activation neurofeedback, which is known to be relevant in terms of cognitive performance enhancement [37].

\section{THE REALISM ARGUMENT}

When compared to various sorts of feedback previously reported in neurofeedback, such as gauges, thermometers [5], colour blocks [15], the initial and naive rationale for the use of virtual agents derives from an attempt at providing realistic visuals. However, this notion of realism needs to be clarified, regardless of any intrinsic limitations due to uncanny effects. We will consider that realistic effects are split alongside several dimensions, which include task realism, social realism and affective realism. We shall only discuss the latter two, since this research does not incorporate task learning or simulation.

When considering neurofeedback experiments designed primarily around emotional responses and emotion regulation, recourse to virtual characters, unlike symbolic feedback displays are relevant to the entire NF loop: they represent a value for the variable the user is trying to regulate, while their baseline state is able to induce emotion as well. This sheds a particular light on the neurofeedback process itself since positive or negative trends may be further amplified by the direct impact of characters' appearance on the very variables that the user is trying to control. An abstract representation, such as a gauge or thermometer, does convey abstract feedback on user performance: this may impact on the users' internal state through their attentional mechanisms, but in a way which largely ignores the natural meaning of the situation represented.

Social realism can be described as the truthful representation of a real-world social situation known to elicit emotional reactions from participants or spectators alike. Social situations can elicit user emotions, as a participant through appraisal mechanisms but also as an observer. The latter is of particular interest in conjunction with some potential neural mechanisms [3]. Affective realism has to do with emotions perceived from the characters' themselves. Modern animation techniques, now available in generic software such as game engines, support a level of facial animation compatible with the expression of easily recognised emotions, which can be further enhanced by associated body attitudes. Transitioning between facial expressions can also be implemented as a neurofeedback channel (see below): whenever the application embedding neurofeedback involves affective rapport with the agent, using agent expressions as a feedback channel is a natural way to design a consistent control loop.

One topic which has attracted little attention so far is the relationship between visual realism and the subject's cognitive strategies for neurofeedback. It seems logical to consider that whenever a realistic character is used for neurofeedback, it could influence the user's cognitive strategies and bias them towards either rapport or communication with that virtual character. Few works have documented the user's declared strategies to sustain NF to the exception of [17] and [28]. User debriefing following our own experiments [11] appears consistent with some of the strategies previously reported and despite the small scale of our sample, a significant proportion of users made reference to communicating with virtual characters in both applications reported below.

\section{EMPATHIC INTERACTION WITH VIRTUAL ACTORS}

An important area of research in virtual agents has been the study of relationships between humans and agents, in particular how human users respond to agents characteristics, perceived for instance as personality traits. Gratch et al. [12] have discussed rapport, and de Melo et al. [9] have discussed moral emotions 


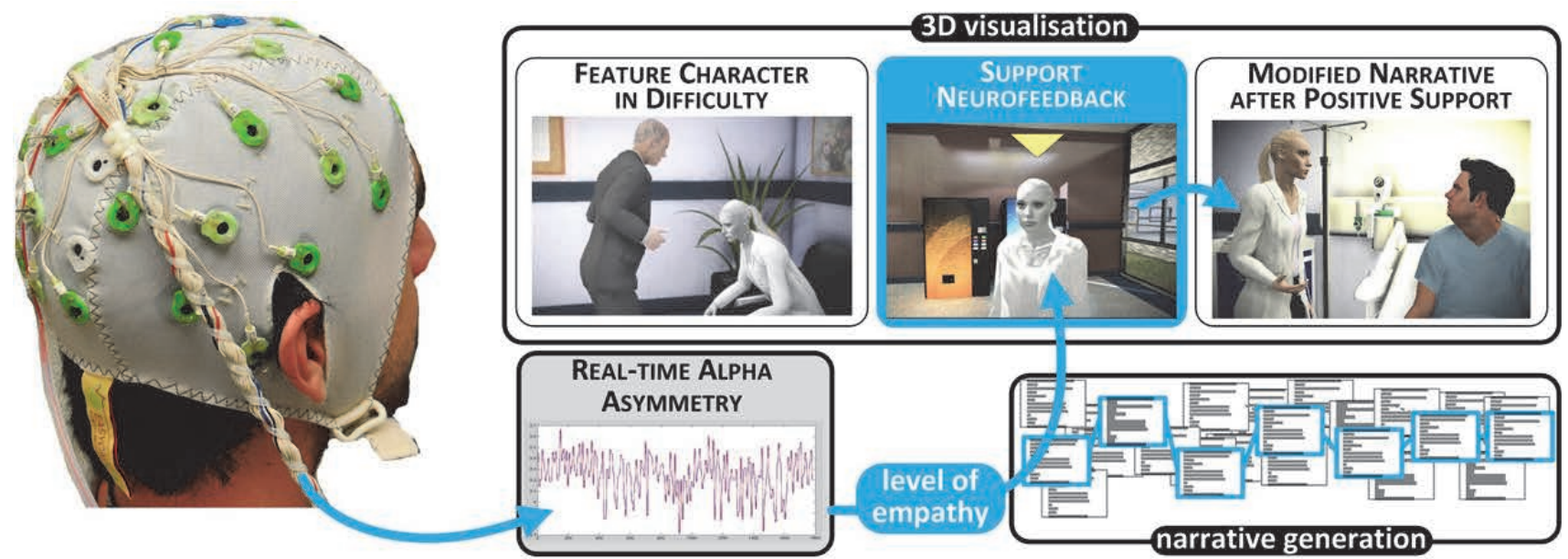

Figure 1. System Overview and Experimental Setting: the user watches the 3D narrative generated in real-time; BCI input (prefrontal alpha asymmetry) during Neurofeedback sessions is interpreted in terms of empathy towards the feature character.

with virtual agents. Morrison and Ziemke [26] have discussed the conditions under which computer games characters can elicit empathic reactions from humans: their perspective was very much a motor one, in line with the hypothesised role of mirror neurons in certain types of empathy. This body of work suggests that agents can elicit attachment, which should make users to respond to further real-time evolution of an agent situation, and/or the expression by the agent of its response to it. From a different perspective, research in filmic theory of emotions has suggested that empathy towards (human) feature characters was a key determinant of viewers' affective response [34].

We have explored the use of an "empathic BCI" for Interactive Narratives featuring virtual actors [11]. Our objectives were to extend affective input in interactive storytelling to incorporate hypotheses from affective filmic theories [34], and to try to reconcile affective input and active interaction, as we had previously explored passive detection of emotional response [10] and active speech-based emotional input [6]. This experiment was also an effort in unifying the different components of the interactive narrative experience around the concept of empathy: narrative generation will be geared towards creating difficult situations for the feature character; empathy will in turn determine the user's emotional response to that situation, and the BCI input will attempt to detect user support, using brain signals previously associated with empathy [19] and amenable to neurofeedback [2, 8]

The baseline story is adapted from the medical drama genre and features a female junior doctor facing multiple difficulties in her professional life. We have previously used the same setting to explore affective response through physiological sensing [10], as the genre is prone to elicit a wide range of affective responses based on the medical situations themselves (visuals and tension), as well as the characters' relationships. The system's architecture is represented in Figure 1. Visual generation and staging takes the form of a machinima-like 3D animation, and is based on the Unreal ${ }^{\circledR}$ (UDK) game engine, which includes support for expressive animations and camera placement. This allows a significant level of dramatization and enhances the potential for empathising with the main character. A baseline set of narrative actions has been defined following the traditional conventions of the medical drama genre: typically one episode will intertwine medical challenges (solving a difficult clinical case) and strained relationships with colleagues, friends and the hierarchy. Each action involving or affecting the feature character is categorised according to the level of hardship it is placing on her. Narrative generation is based on landmark planning [30] and is biased towards placing the character through difficult situations. Although the system will generate a range of different stories, they all have in common her demise as the story ending (e.g. medical malpractice, breakdown, etc.), as the story builds up adverse situations and difficulties. The system is biased towards creating difficult situations at an early stage (first third of the story). Once the character is faced with a serious situation, she will turn to the user for support. This manifests itself through an embedded neurofeedback session, the user having been briefed about the possibility of helping her by "expressing positive thoughts". Should the user succeed in supporting her, the remainder of the narrative will evolve in a direction more favourable to the character. The user is given between one and two opportunities for interaction, which take the form of short $30 \mathrm{~s}$ neurofeedback sessions. The neurofeedback technique consists in a measure of pre-frontal alpha EEG asymmetry using Davidson's a2 score [7]. This measure is correlated with approach/avoidance, as well as a specific kind of empathy described as cheerful empathy [19].

The neurofeedback session is triggered by interrupting the narrative action and transitioning to a cut scene in which the character stands alone in the hospital corridor, facing the user. The level of desperation of the character is visualised by desaturating its colour until it appears in black and white within an otherwise colour scene (Figure 2). This neurofeedback session can be termed non-diegetic since it interrupts the natural flow of the

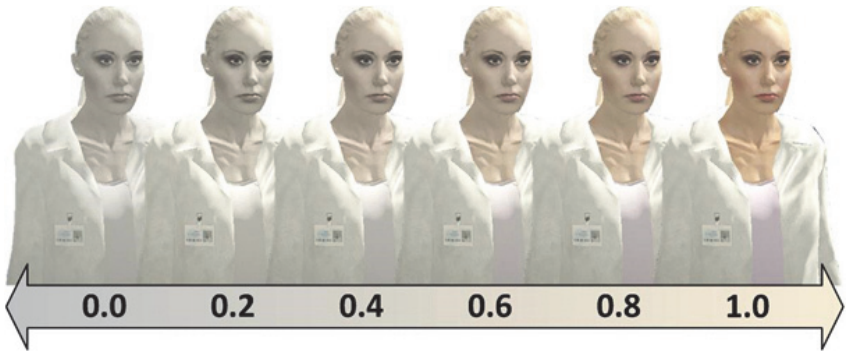

Figure 2. Feedback channel for the filmic empathy experiment. When in need of support, the feature character would fade to grey. During Neurofeedback, alpha asymmetry scores are mapped onto the character's colour saturation. 


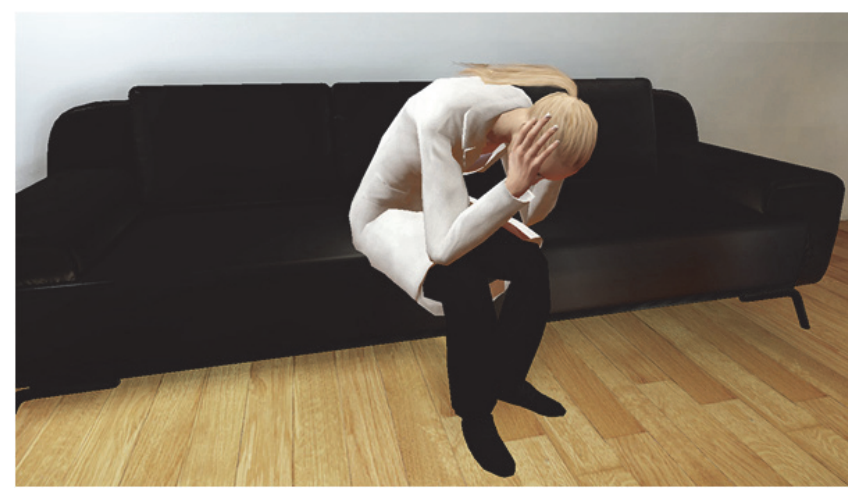

Figure 3. The feature character facing a difficult situation.

narrative, as well as departing from visual realism. Another justification to isolate the NF session is the hypothesised difficulty for users to sustain the BCI input by altering their pre-frontal activation. The role of the virtual character in the NF process is thus double: it elicits empathic responses by expressing its disarray (Figure 3) and it serves as the output signal for neurofeedback.

We carried out a first set of experiments in which subjects were using the system inside an fMRI scanner (using an MRIcompatible EEG device/recorder) to provide an empirical validation that successful NF sessions were indeed associated to an asymmetry in pre-frontal areas. Subjects were introduced to the interactive narrative and explained that they would be able to influence it by supporting the feature characters "through positive thinking", without being given explicit instructions. They were familiarised with the colour-saturation neurofeedback approach through short training sessions outside the MRI scanner. Out of twelve subjects, six were successful in their NF session and in altering the course of action of the narrative. Successful subjects showed various levels of asymmetry in the pre-frontal cortex compared to a control condition that consisted in passively viewing a replay of the generated story without NF. No differences were observed in other areas, in particular motor areas. These findings are consistent with our original hypothesis as well as with post-experiment debriefings in which subjects described their cognitive strategies for neurofeedback, which showed a bias towards explicit strategies [11]. The 50\% success score may appear only average, but it should be interpreted considering previous reports of up to $30 \%$ 'BCI illiteracy' [35] (the inability of a subject population to engage in NF) the difficult environment constituted by the fMRI scanner, and most importantly the limited duration of training (one pre-experiment session of 10 minutes, compared to repeated sessions totalling several hours for most pre-frontal alpha-asymmetry reported work).

The choice of non-diegetic feedback was initially dictated by the need to isolate the neurofeedback sessions within the course of the interactive narrative, taking into account the cognitive effort required from the subject. Our initial choice was towards a visual mapping philosophy, which supported continuous quantifiable feedback. Yet, despite the positive results obtained during the above experiments, one criticism sometimes made was the unrealistic nature of the interaction and display. This has led us to explore the possibility of implementing NF through a more diegetic approach, one in which the character's situation and appearance would be compatible with the staging of the narrative.

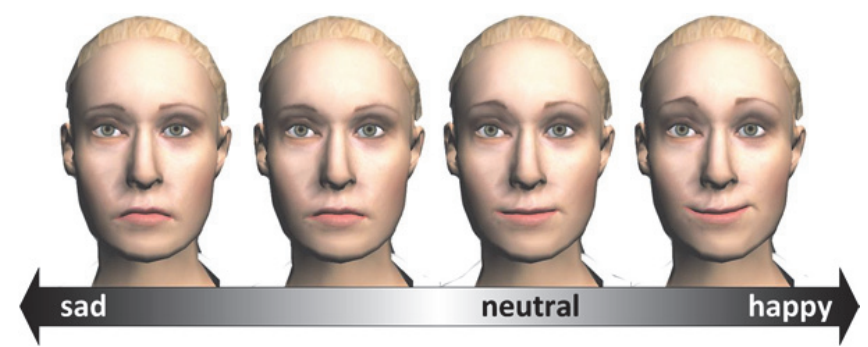

Figure 4. Diegetic neurofeedback takes the form of real-time transitions between facial expressions.

As a consequence of the effort-related constraints, the neurofeedback session will still have to be well-defined and segregated from the main course of action, but this segregation should be based on narrative principles. This means that the character will have to isolate herself, for instance by locking herself in the restroom to cry, with a corresponding transition in terms of narrative actions. The main challenge in developing diegetic NF at the visual level is to find appropriate display rules still centred on the character (we have ruled out sound-based or atmospheric feedback, such as lighting intensity or shades, as disrupting the empathic triangle). The natural approach is to display the emotional state of the agent through non-verbal behaviour, using continuous variations that can be the target of neurofeedback mapping. The emotional state can be represented through a combination of facial expressions and body attitudes, although the main challenge is to devise a believable continuum between opposite states in terms of valence. Facial expressions have been dedicated significant work in terms of parameterisation for recognition and generation alike, in particular through Facial Animation Parameters (FAP), which are now supported by most animation systems including those embedded in the Unreal ${ }^{\circledR}$ (UDK) game engine we have been using. FAP descriptions are available for basic emotions such as sadness and happiness, which would make it possible to consider neurofeedback output to be based on a transition between sadness and happiness. From a diegetic perspective this would materialise NF-based user support as succeeding in comforting the character. An additional challenge for NF realism is however that little work has been dedicated to the transitioning between opposite facial expressions: Stoiber et al. [32] have introduced a system to control facial emotions based on dimensional models, offers an efficient realtime transition path between any two expressions, however based on interpolation techniques that may not always be consistent with real-world phenomena. We have thus designed an ad hoc FAPbased mapping between the expressions of sorrow and happiness, however transitioning through neutral expressions (Figure 4); the acceptability of more steep transitions in terms of neurofeedback would be a matter for further experimentation.

\section{VIRTUAL CROWDS AND EMOTION REGULATION}

In the previous sections we have reported emotion elicitation and expression by an individual character. One less developed area of research is to consider that populations of virtual agents, or virtual crowds, rather than individuals, can be used for the purpose of emotion elicitation [24], considering the same basic emotions as those expressed by an individual agent. One major difference rests with the fact that the study of user-crowd interaction is much less developed, in particular for virtual crowds, as these tend to be observed by virtual humans from an external perspective. 


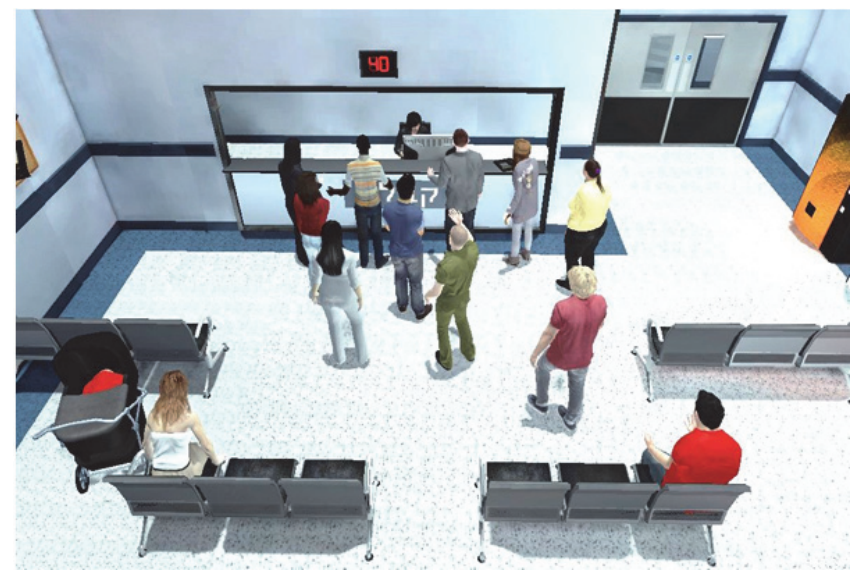

Figure 5. Overview of the Waiting Room environment.

In this second set of experiments, we have explored crowd behaviour as a potential backchannel for NF. The objective of this research was to explore the impact of realistic, as opposed to abstract, visual feedback, in the context of more basic research in fMRI or EEG-based NF. While our previous work was dedicated to incorporating NF-based BCI into an interactive storytelling system, this one is devoid of any media component, and was instead designed to support NF research.

Several questions are attached to this approach. The first one is to revisit the alignment between brain areas tracked by NF, and the type of emotions conveyed by the agents. The second one concerns the temporal aspects of visual feedback and how they would influence the overall NF design paradigm.

It is not sufficient for a virtual crowd simulation to be realistic; it also needs to correspond to a believable situation. We have thus designed a virtual environment in which a population of agents could rapidly evolve between emotional states in a realistic context. The situation recreated is the waiting room of a hospital, populated by twenty virtual agents playing the role of patients or family members, and one single agent staffing the reception desk (Figure 5). Here virtual agents are used not individually, but as a crowd. The emotional signal visualised through the crowd behaviour corresponds to the arousal dimension, which is represented by the level of agitation of the crowd. In the waiting room environment, this corresponds to the number of agents standing up at the desk and protesting about the waiting time. The brain region tracked in our NF experiments is precisely the amygdala, whose role and level of activation are generally associated with arousal [21]. Hence the "virtual" arousal of the crowd, which is the matching variable, is used to visualise the real arousal level of the subject controlled through her amygdala activity.

This experiment (Figure 6) is also based on a NF paradigm, and comprises a target emotional response (amygdala activity), an input probe (amygdala EEG Fingerprint), and a mapping to an "unrest" metric that governs the global behaviour of the visual environment. Once again, appropriate user instructions are issued to the user so she can follow the NF paradigm. The overall experiment investigates the control of arousal based on down- or up-regulation of the amygdala. The sensor is a bespoke EEG fingerprint (EFP) of the amygdala activity obtained from fMRI data, consisting in a nonstandard electrode placement as described in [25]. In terms of signal acquisition, it brings flexibility, ease of use and specificity to the target region. It allows a response time, which is that of the EEG signal, making it possible to design appropriate feedback loops not limited by signal acquisition delays.

The system is implemented using the Unreal ${ }^{\circledR}$ (UDK) game engine: agents' behaviours are represented through various features of the engine: idle animations, motion planning and expressive animations. Each corresponds to a different component of crowd expressivity. Idle animations are required to ensure realism of the sat down fraction of the agent population, yet its parameterisation can convey a first level of unrest amongst the crowd. Motion planning, including corresponding animations is the main mechanism for crowd behaviour, in that it controls the ratio between characters waiting and characters standing at the front desk. Expressive animations that correspond to argumentative conversation further enhance the realism of the standing part of the crowd. In addition, the sound features of the Unreal ${ }^{\circledR}(\mathrm{UDK})$ game engine have been used to playback a global soundtrack in the form of loud conversations, whose intensity can be tuned to the unrest level of the crowd.

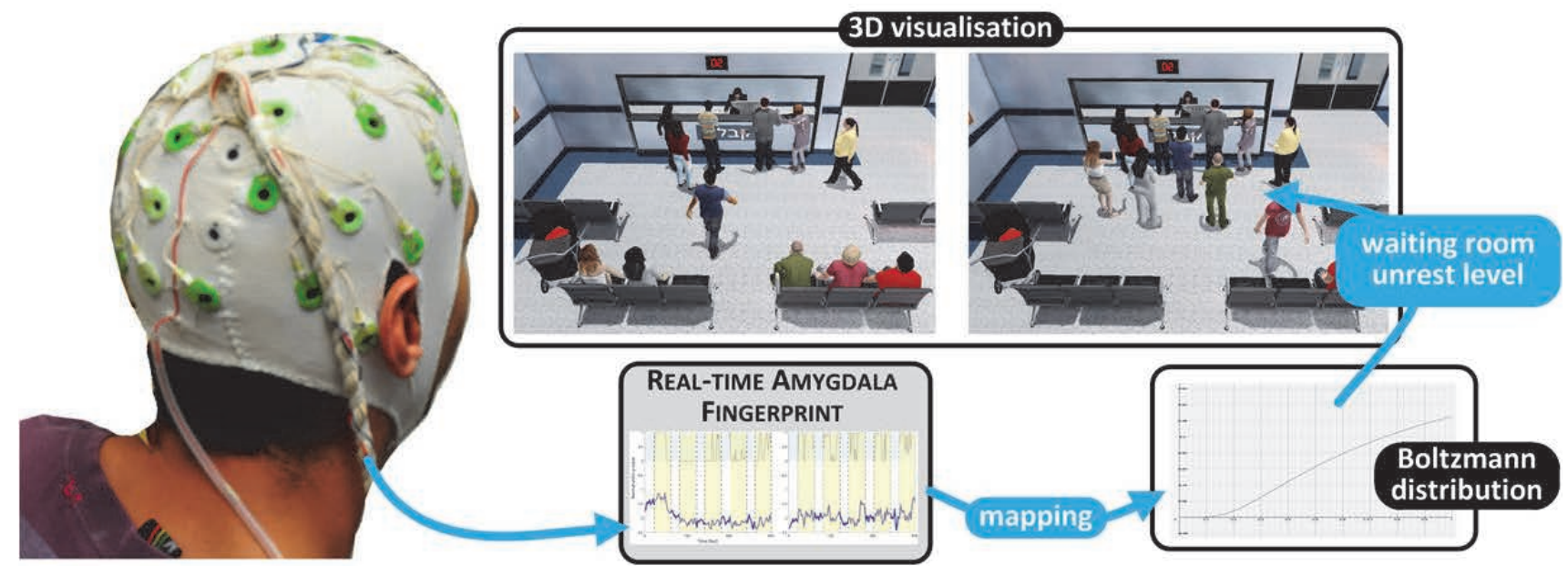

Figure 6. System Overview and Experimental Setting: the user is instructed to either appease the situation in the waiting room, or to foster unrest. Neurofeedback is based on a bespoke EEG Fingerprint for the amygdala: the level of unrest in the waiting room matches the subject's amygdala's activation. 


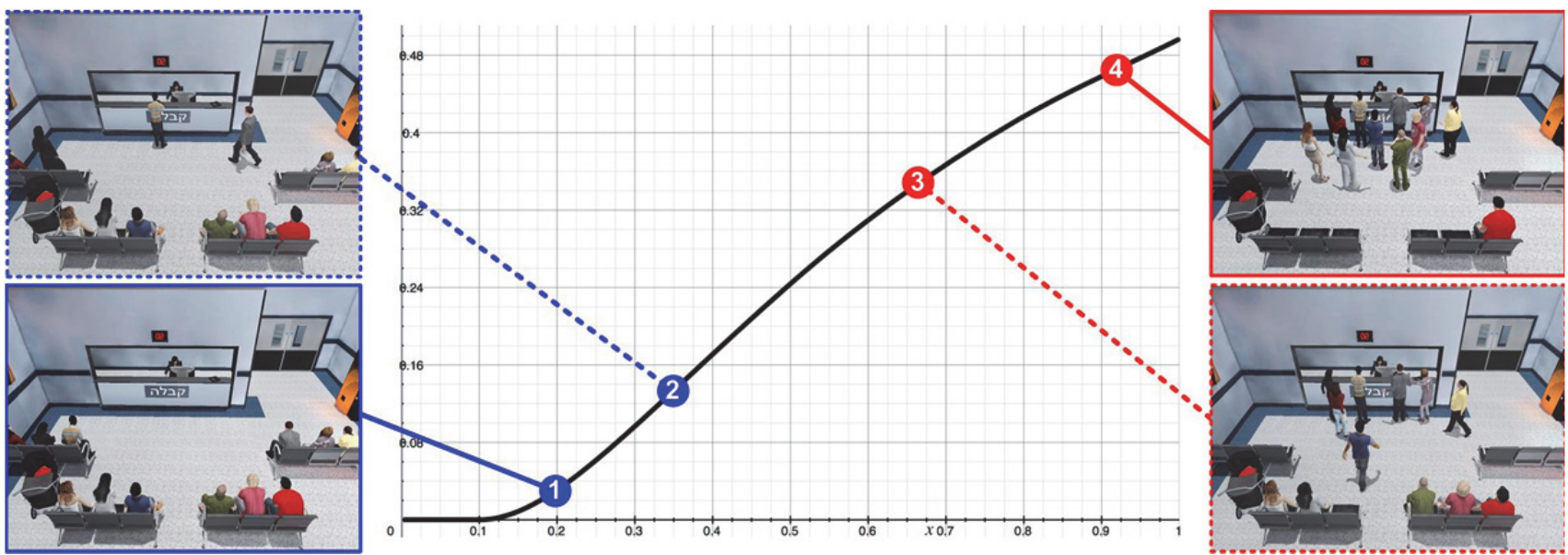

Figure 7. Levels of unrest in the waiting room derived from the amygdala activity using a Boltzmann distribution.

As part of the NF loop, we need to be able to consistently control the overall behaviour of the crowd with a global variable. To that effect, we consider the global crowd behaviour to be described by the distribution between resting and agitated characters, which is best described by a statistical model. By analogy with statistical models describing the transition of particles between energy levels, we use a Boltzmann-type distribution for which the two levels of energy correspond to characters resting, or protesting at the front desk. The controlling variable is represented by the temperature $\mathrm{T}$ in Boltzmann's equation, which is used as determining the global level of unrest in the waiting room. The NF mapping is defined by using the measured amygdala activity as an analogue of temperature in the Boltzmann equation. The other parameters in the equation, such as energy levels, have been determined empirically by a calibration step, which adapts the target distribution of agents to the variation range of the amygdala EFP input signal. In terms of NF cycles, at each step the EFP signal is acquired and converted into a virtual temperature in the Boltzmann equation (Figure 7). This is used to update accordingly the ratio between quiet and agitated characters, which is then adjusted in the waiting room by computing the required adjustment between number of characters in each state, and randomly selecting agents for transition. Transitioning from states is visualised in terms of the agent standing up from its seat and reaching the counter or, conversely returning to its initial seat. While the change in state for an individual agent requires a few seconds to be completed (depending on its distance from the front desk), the directions in which agents are moving is clearly visible.

Six volunteers ( 5 male, 1 female) participated in our preliminary experiment. The EEG signal was acquired using a BrainAmp-MR EEG amplifier (Brain Products, Munich, Germany) and the BrainCap electrode cap with $\mathrm{Ag} / \mathrm{AgCl}$ electrodes providing 30 EEG channels, and additional ECG and EOG channel (Falk Minow Services, Herrsching-Breitbrunn, Germany). The EEG signal was sampled at $5 \mathrm{kHz}$ and recorded using the brain vision recorder software (Brain Products).

The EEG fingerprint of the amygdala was computed as described in [25]. It was sampled continuously during a 5 minutes baseline session. The participants were instructed to sit still while no external stimuli were introduced to them. The EFP computed during this baseline session was used for standardising data collected during the next NF sessions. During these NF sessions, the standard score of the EFP relative to the baseline distribution served as a target for the NF manipulation.

Each of the four NF sessions alternates four baseline epochs of passive viewing $(30 \mathrm{sec})$ with four blocks of active NF $(60 \mathrm{sec})$. In the first two sessions the participants were instructed to restore order in the waiting room using their brain activity; in the latter two they were instructed to foster unrest. Once again, users were not given more specific advice or instructions to avoid suggesting explicit strategies. In the last two sessions, the participants were asked to foster unrest in the waiting room (the initial distribution of characters can be parameterised depending on the session's objective given to the subject).

Overall, participants were successful in at least one of their NF blocks, and most of them over several. Figure 8 displays results for one subject over neurofeedback blocks on a task which consisted in up-regulating the amygdala.

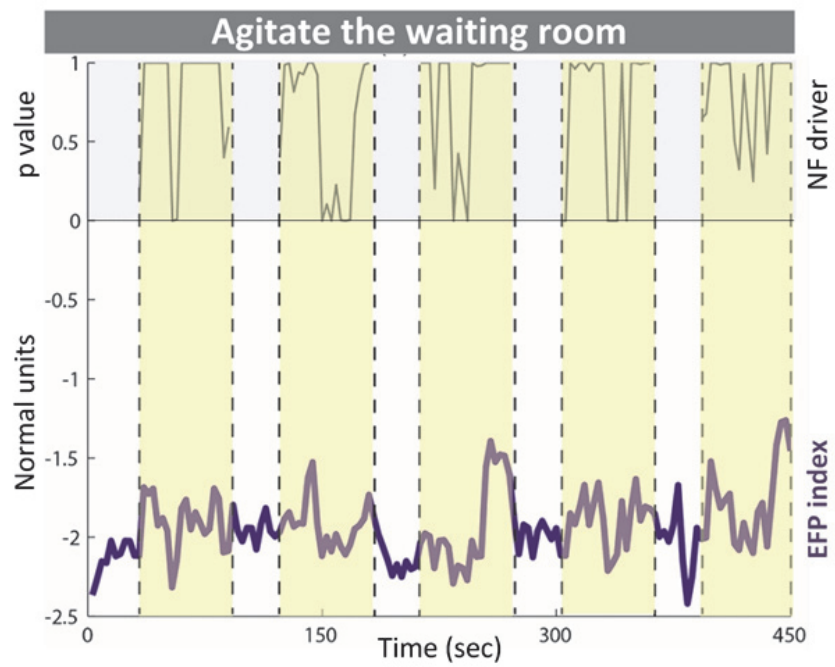

Figure 8. Neurofeedback session for amygdala up-regulation. The amygdala activity is plotted during several short Neurofeedback trials (the top part of the curve plots the statistical significance of Neurofeedback success). 


\section{CONCLUSION}

An increasing number of BCI systems are turning to virtual agents as an interface displaying NF signals. Depending on the main focus of the application, agents can: i) support various metaphors (such as the pole vault jump of [14]), ii) participate to the realism of the situation the subjects are training for (e.g. a classroom in ADHD treatment applications), or iii) constitute the main target of the application (e.g., in gaming or interactive narrative).

It is probably too early to draw generic conclusions about the potential benefits of virtual agents, outside those applications where they already feature prior to the adoption of $\mathrm{BCI}$ techniques. However, our preliminary results suggest that virtual agents can successfully support neurofeedback applications in affective BCI or emotional regulation experiments. This extends the research programme introduced in [16], which suggested that more sophisticated visualisation could improve neurofeedback performance. On the other hand, this is somehow in contradiction with the previous assumption that neurofeedback should be based on a simple and direct representation of the target parameters values [28].

More systematic research is required to compare the usability of neurofeedback systems using abstract displays with those featuring virtual agents, exploring important aspects such as temporal behaviour, ease of training and its impact on BCI illiteracy scores [35]

\section{ACKNOWLEDGEMENTS}

We thank Catherine Pelachaud and Radoslaw Niewiadomski for insightful comments on the dynamics of facial expressions.

\section{REFERENCES}

[1] Atkins, P. and De Paula, J. 2006. Atkins' physical chemistry. Oxford University Press, Oxford.

[2] Baehr, E., Rosenfeld, J.P., and Baehr, R. 2001. Clinical use of an alpha asymmetry neurofeedback protocol in the treatment of mood disorders: Follow-up study one to five years post therapy. Journal of Neurotherapy 4, 4, 11-18.

[3] Blair, R. 2007. The amygdala and ventromedial prefrontal cortex in morality and psychopathy. Trends in Cognitive Sciences 11, 9, 387-392.

[4] Bos, D.P., Reuderink, B., van de Laar, B., Gürkök, H., Mühl, C., Poel, M., Nijholt, A., and Heylen, D. 2010. Braincomputer interfacing and games. In Brain-Computer Interfaces, D. Tan and A. Nijholt, Eds. Springer, 149-178.

[5] Caria, A., Veit, R., Sitaram, R., Lotze, M., Weiskopf, N., Grodd, W., and Birbaumer, N. 2007. Regulation of anterior insular cortex activity using real-time fMRI. NeuroImage 35, 3, 1238-1246.

[6] Cavazza, M., Pizzi, D., Charles, F., Vogt, T., and André, E. 2009. Emotional input for character-based interactive storytelling. In Proceedings of the 8th International Conference on Autonomous Agents and Multiagent SystemsVolume 1, International Foundation for Autonomous Agents and Multiagent Systems, 313-320.

[7] Davidson, R.J. 1992. Anterior cerebral asymmetry and the nature of emotion. Brain and Cognition 20, 1, 125-151.

[8] Davidson, R.J. 2004. What does the prefrontal cortex "do" in affect: perspectives on frontal EEG asymmetry research. Biological Psychology 67, 1, 219-234.
[9] de Melo, C.M., Zheng, L., and Gratch, J. 2009. Expression of moral emotions in cooperating agents. In Intelligent Virtual Agents, Zs. Ruttkay, M. Kipp, A. Nijholt, H. Vilhjálmsson, Eds. Springer, 301-307.

[10] Gilroy, S., Porteous, J., Charles, F., and Cavazza, M. 2012. Exploring passive user interaction for adaptive narratives. In Proceedings of the 2012 ACM international conference on Intelligent User Interfaces, ACM, 119-128.

[11] Gilroy, S.W., Porteous, J., Charles, F., Cavazza, M., Soreq, E., Raz, G., Ikar, L., Or-Borichov, A., Ben-Arie, U., and Klovatch, I. 2013. A brain-computer interface to a plan-based narrative. In Proceedings of the Twenty-Third International Joint Conference on Artificial Intelligence, AAAI Press, 1997-2005.

[12] Gratch, J., Wang, N., Gerten, J., Fast, E., and Duffy, R. 2007. Creating rapport with virtual agents. In Intelligent Virtual Agents, C. Pelachaud, J-C. Martin, E. André, G. Chollet, K. Karpouzis, D. Pelé, Eds. Springer, 125-138.

[13] Gruzelier, J.H. 2013. EEG-neurofeedback for optimising performance. I: A review of cognitive and affective outcome in healthy participants. Neuroscience \& Biobehavioral Reviews, in press.

[14] Heinrich, H., Gevensleben, H., Freisleder, F.J., Moll, G.H., and Rothenberger, A. 2004. Training of slow cortical potentials in attention-deficit/hyperactivity disorder: evidence for positive behavioral and neurophysiological effects. Biological Psychiatry 55, 7, 772-775.

[15] Huster, R.J., Mokom, Z.N., Enriquez-Geppert, S., and Herrmann, C.S. 2013. Brain-computer interfaces for EEG neurofeedback: Peculiarities and solutions. International Journal of Psychophysiology 91, 1, 36-45.

[16] Jensen, C.B.F., Petersen, M.K., Larsen, J.E., Stopczynski, A., Stahlhut, C., Ivanova, M.G., Andersen, T., and Hansen, L.K. 2013. Spatio temporal media components for neurofeedback. In Proceedings of the 2013 IEEE International Conference on Multimedia and Expo (ICME).

[17] Kober, S.E., Witte, M., Ninaus, M., Neuper, C., and Wood, G. 2013. Learning to modulate one's own brain activity: the effect of spontaneous mental strategies. Frontiers in Human Neuroscience 7, Article 695.

[18] Lécuyer, A., Lotte, F., Reilly, R.B., Leeb, R., Hirose, M., and Slater, M. 2008. Brain-computer interfaces, virtual reality, and videogames. Computer 41, 10, 66-72.

[19] Light, S.N., Coan, J.A., Zahn-Waxler, C., Frye, C., Goldsmith, H.H., and Davidson, R.J. 2009. Empathy is associated with dynamic change in prefrontal brain electrical activity during positive emotion in children. Child Development 80, 4, 1210-1231.

[20] Lim, C.G., Lee, T.S., Guan, C., Fung, D.S.S., Zhao, Y., Teng, S.S.W., Zhang, H., and Krishnan, K.R.R. 2012. A Brain-Computer Interface Based Attention Training Program for Treating Attention Deficit Hyperactivity Disorder. PloS one 7, 10, e46692.

[21] Lindquist, K.A., Wager, T.D., Kober, H., Bliss-Moreau, E., and Barrett, L.F. 2012. The brain basis of emotion: A metaanalytic review. Behavioral and Brain Sciences 35, 3, 121143. 
[22] Lugrin, J. and Cavazza, M. 2006. AI-based world behaviour for emergent narratives. In Proceedings of the 2006 ACM SIGCHI International Conference on Advances in Computer Entertainment Technology, ACM, 25.

[23] Marshall, D., Coyle, D., Wilson, S., and Callaghan, M. 2013. Games, gameplay, and BCI: The state of the art. IEEE TCIAIG 5, 2, 82-99.

[24] McHugh, J.E., McDonnell, R., O'Sullivan, C., and Newell, F.N. 2010. Perceiving emotion in crowds: the role of dynamic body postures on the perception of emotion in crowded scenes. Experimental Brain Research 204, 3, 361372.

[25] Meir-Hasson, Y., Kinreich, S., Podlipsky, I., Hendler, T., and Intrator, N. 2013. An EEG Finger-Print of fMRI deep regional activation. NeuroImage, in press.

[26] Morrison, I. and Ziemke, T. 2005. Empathy with computer game characters: A cognitive neuroscience perspective. In Proceedings of the Joint Symposium on Virtual Social Agents, AISB, Hatfield, U.K., 73-79.

[27] Mühl, C., Brouwer, A., van Wouwe, N., van den Broek, E., Nijboer, F., and Heylen, D. 2011. Modality-specific affective responses and their implications for affective BCI. In Proceedings of the Fifth International Brain-Computer Interface Conference, G.R. Müller-Putz, R. Scherer, M. Billinger, A. Kreilinger, V. Kaiser, C. Neuper, Eds. Verlag der Technischen Universität, Graz, Austria, 120-123.

[28] Nan, W., Rodrigues, J.P., Ma, J., Qu, X., Wan, F., Mak, P., Mak, P.U., Vai, M.I., and Rosa, A. 2012. Individual alpha neurofeedback training effect on short term memory. International Journal of Psychophysiology 86, 1, 83-87.

[29] Niv, S. 2013. Clinical efficacy and potential mechanisms of neurofeedback. Personality and Individual Differences 54, 6, 676-686.
[30] Porteous, J., Cavazza, M., and Charles, F. 2010. Applying planning to interactive storytelling: Narrative control using state constraints. ACM Transactions on Intelligent Systems and Technology (TIST) 1, 2, 1-21.

[31] Renard, Y., Lotte, F., Gibert, G., Congedo, M., Maby, E., Delannoy, V., Bertrand, O., and Lécuyer, A. 2010. OpenViBE: an open-source software platform to design, test, and use brain-computer interfaces in real and virtual environments. Presence: Teleoperators and Virtual Environments 19, 1, 35-53.

[32] Stoiber, N., Seguier, R., and Breton, G. 2009. Automatic design of a control interface for a synthetic face. In Proceedings of the 14th international conference on Intelligent User Interfaces, ACM, 207-216.

[33] Sulzer, J., Haller, S., Scharnowski, F., Weiskopf, N., Birbaumer, N., Blefari, M., Bruehl, A., Cohen, L., Decharms, R., and Gassert, R. 2013. Real-time fMRI neurofeedback: Progress and challenges. NeuroImage 76, 386-399.

[34] Tan, E.S. 1996. Emotion and the structure of narrative film: Film as an emotion machine. Lawrence Erlbaum Associates, Mahwah, N.J.

[35] Vidaurre, C. and Blankertz, B. 2010. Towards a cure for BCI illiteracy. Brain Topography 23, 2, 194-198.

[36] Weiskopf, N. 2012. Real-time fMRI and its application to neurofeedback. NeuroImage 62, 2, 682-692.

[37] Zoefel, B., Huster, R.J., and Herrmann, C.S. 2011. Neurofeedback training of the upper alpha frequency band in EEG improves cognitive performance. NeuroImage 54, 2, $1427-1431$. 